\title{
¿Conflictos en la
}

integración regional?

Consideraciones acerca de la superposición de los acuerdos internacionales de inversión, los acuerdos comerciales regionales y la $\mathrm{OMC}^{1}$

\author{
ANDREAS R. ZIEGLER ${ }^{2}$ \\ LAINA P. MAIA ${ }^{3}$
}

\section{RESUMEN}

La creciente interdependencia económica internacional resultó en el desarrollo de diversas organizaciones y acuerdos regionales como los acuerdos comerciales regionales, los acuerdos internacionales de inversión y la Organización Mundial de Comercio (OMC). En el panorama actual, diversos países son miembros de estos acuerdos y organizaciones concomitantemente. El gran incremento de los resultados de soluciones de diferencias debido al éxito del Entendimiento sobre Solución de Diferencias (ESD) de la OMC y el crecimiento paralelo del uso de las provisiones de los All sobre solución de diferencias entre Estados e inversores llevaron a afirmaciones sobre el distanciamiento del derecho internacional de las inversiones y el derecho internacional comercial, lo que podría generar problemas con relación a la coherencia y estabilidad del derecho económico

1 Este documento fue elaborado en el marco del Seminario SLADI 2015. Para citar el artículo: ZiEGLER, A. \& MAIA, L. (2016). ¿Conflictos en la integración regional? Consideraciones acerca de la superposición de los acuerdos internacionales de inversión, los acuerdos comerciales regionales y la OMC, en Revista Con-Texto, n. ${ }^{\circ} 46$ EE, pp. 77-97. DOI: https://doi.org/10.18601/01236458.n46EE.05

2 Catedrático de Derecho y director del Programa de LLM en Derecho Económico y Comercial Internacional y Europeo de la Facultad de Derecho de la Universidad de Lausana (Suiza). Profesor asociado de la Facultad de Derecho de Nueva Gales del Sur (Australia). Profesor visitante permanente del Instituto Suizo Federal de Tecnología (ETH) de Zúrich y de la Universidad de St. Gallen (Suiza). Presidente de la Asociación de Derecho Internacional - ILA Suiza. Correo electrónico: andreas.ziegler@unil.ch

3 Consultora de la División de Inversión y Empresa de la CNUCED. Maestría en Derecho Económico, Universidad Federal de Paraíba, Brasil. Máster en Derecho de la Unión Europea, Universidad Carlos III de Madrid, España. Doctoranda en Derecho Internacional en la Universidad de Lausana, Suiza. Correo electrónico: laina.maia@unil.ch 
internacional. La doctrina afirma la existencia de un "spaghetti bowl" de las provisiones de solución de diferencias de los acuerdos comerciales regionales, los acuerdos internacionales de inversión, la OMC y la jurisprudencia resultante de estos. El presente artículo propone un análisis de las disposiciones superpuestas sobre solución de controversias en los acuerdos económicos internacionales, observando las interacciones de las disposiciones de la OMC y del MERCOSUR con respecto al tema. Por fin, se elaboran consideraciones sobre la necesidad de mejorar los mecanismos institucionales, para aumentar la coherencia tanto en el ámbito de la inversión como en el del comercio.

Palabras clave: Integración regional. Acuerdos comerciales regionales. Acuerdos internacionales de inversión. Superposición. MERCOSUR.

\section{INTRODUCCIÓN}

A pesar de tener orígenes comunes, el régimen internacional de inversiones y el régimen internacional o mundial comercial se han desarrollado separadamente durante mucho tiempo. Esta característica se tornó bastante evidente cuando la Organización Mundial de Comercio (OMC) comenzó sus actividades en enero de $1995^{[4]}$. Acuerdos internacionales de inversiones (AII) bilaterales y regionales, principalmente, continuaron siendo firmados, mientras los intentos de negociación de un acuerdo multilateral sobre inversiones $(\mathrm{AMI})^{5}$ en el seno de la Organización para la Cooperación y Desarrollo Económico (OCDE) fallaron en definitivo en 1998. El gran aumento en el número de soluciones de controversias debido al éxito del Entendimiento sobre Solución de Diferencias (ESD) de la OMC y el crecimiento paralelo del uso de las provisiones de los AII sobre solución de diferencias inversionista - Estado llevaron a afirmaciones sobre el distanciamiento de las dos áreas del derecho económico internacional, lo que podría generar problemas con relación a la coherencia y estabilidad de este área del derecho ${ }^{6}$.

¿Pero el actual "spaghetti bowl" de las provisiones de solución de diferencias de los acuerdos comerciales regionales (ACR), los AII, la OMC y su jurisprudencia resultante demandan acciones más concretas? ${ }^{7}$ Gran parte de la doctrina reciente sobre inversiones

4 Sobre el desarrollo de la OMC y sus características principales, véase: QURESHI, ASIF y ZIEGLER, ANDREAS R. International Economic Law. 3 ed., Londres: Sweet and Maxwell, 2011, p. 309, y ZIEGLER, ANDREAS R. Droit international économique de la Suisse. Berne, 2009, p. 111.

5 Sobre la historia del AMI, véase QuRESHI y ZIEGLER. Op. cit, p. 492.

6 Sobre los respectivos sistemas de solución de diferencias, véase QuRESHI y ZIEGLER. Op. cit, p. 1, 430 y 522 .

7 Por supuesto, esta cuestión también puede ser vista como parte del debate aún más complejo sobre la fragmentación del orden jurídico internacional y la (aparente) fragmentación del derecho internacional público. Véase, por ejemplo, FauChald, Kristian y NollKaEMPER, André (eds.). The Practice of International and National Courts and the (De-)Fragmentation of International Law. Oxford: Hart Publishing, 2012, o TeITEL, Ruti y Howse, RoberT L. Cross-Judging: Tribunalization in a Fragmented But Interconnected Global Order. New York University Journal of International Law and Politics (JILP). Forthcoming, NYU School of Law, Public Law Research. Paper no. 09-04. NYLS Legal Studies Research. 
estuvo centrada en procedimientos paralelos o incoherentes de soluciones de controversias en el área de los procedimientos entre Estados e inversores. En general, las posibles interferencias de las disposiciones sobre solución de diferencias en AII no han sido analizadas a fondo, incluyendo capítulos sobre inversiones en ACR, sobre el funcionamiento del sistema multilateral de comercio y su sistema de solución de diferencias, así como la creciente estructura paralela de los ACR. Los conceptos clave normalmente asociados a este entendimiento incluyen: forum shopping, requisitos conflictivos, procedimientos paralelos, superposición institucional e interpretación divergente ${ }^{8}$.

Muchos AII y ACR modernos contienen disposiciones sobre solución de controversias inversionista - Estado que están abiertas a los particulares (inversores extranjeros). Estos procedimientos se han utilizado cada vez más en los últimos diez años, y los inversores han sido muy innovadores al utilizarlos para extender su protección frente a un amplio ámbito de las políticas impuestas por los gobiernos. Esta nueva tendencia ha emergido en el Tratado de Libre Comercio de la América del Norte (TLCAN) ${ }^{9}$, con las demandas de inversores que desafiaban las medidas de política comercial de los Estados. Un gran número de reclamaciones en virtud del capítulo 11 del TLCAN estaban relacionadas con las cuestiones comerciales (incluida la contratación pública ${ }^{10}$, derechos compensato$\operatorname{rios}^{11}$, etc.) , y esto también puede pasar en el contexto de los AII y otros ACR que prevén la solución de controversias inversionista - Estado ${ }^{12}$. Algunos autores apuntan que esto

Paper no. 09/10 \#14. Disponible en internet: SSRN: http://ssrn.com/abstract=1334289. Cabe señalar, entre tanto, que todo el tema del asunto 3 (2011) Transnational Dispute Management (TDM), editado por TODD WEILER, fue dedicado a "Intersections: Dissemblance or Convergence between International Trade and Investment Law".

8 Véase Petersmann, Ernst-Ulrich. Justice as Conflict Resolution: Proliferation, Fragmentation and Decentralization of Dispute Settlement in International Trade. EUI Working Paper LAW no. 2004/10, 2004. Disponible en internet: SSRN <http://ssrn.com/abstract=836324>; VAN AAKEN, ANNE. Fragmentation of International Law: The Case of International Investment Protection. 17 Finnish Yearbook of International Law 93, 2008.

9 North American Free Trade Agreement Between the Government of Canada, the Government of Mexico and the Government of the United States [NAFTA], 17 diciembre 1992, Can TS 1994 no. 2, 32 ILM 289 (entrada en vigor, 1 de enero de 1994).

10 ADF Group Inc. v United States of America, Award, ICSID Case n. ${ }^{\circ}$ ARB(AF)/00/1 (9 January 2003); véase también, CJ Michael Flavell QC, Martin G Masse y Cyndee Todgham Cherniak. Buy American or What? International Trade Brief, 2009. Disponible en internet: McMillan <http://www.mcmillan.ca/ Buy-American-or-What>.

11 Además de los famosos casos de madera blanda -infra nota 13 y texto correspondiente-, véase también VoOn, TANIA. NAFTA Chapter 19 Panel Follows WTO Appellate Body in Striking Down Zeroing. 14:29 American Society of International Law, ASIL Insights, 2010. Disponible en internet: ASIL < http://www.asil. org/files/insight100923pdf.pdf>; con relación a los casos: Re Stainless Steel Sheet and Strip In Coils from Mexico (Mexico v United States), USA-MEX-2007-1904-01 (Ch 19 Panel), 2010. Disponible en internet: NAFTA <http://registry.nafta-sec-alena.org/cmdocuments/edce701c-9720-424b-b232-1fd714d318ba. pdf $>$; United States - Final Anti-Dumping Measures on Stainless Steel from Mexico (Complaint by the United States), WTO Doc WT/DS50/AB/R (Appellate Body Report), 2008. Disponible en internet: WTO < http://docsonline.wto.org >.

12 Un ejemplo relacionado con cuestiones sanitarias y fitosanitarias es una disputa que implica al productor de cigarrillos Philip Morris International (PMI), que desafió los requisitos de etiquetado en Uruguay, en 
posibilita a los particulares la impugnación de medidas comerciales ante tribunales arbitrales, sin depender de la buena voluntad de sus Estados para llevar un caso a la solución de diferencias de la OMC o de Estado a Estado en varios ACR (como el capítulo 20 en el TLCAN $)^{13}$.

El presente artículo analizará las disposiciones superpuestas sobre solución de controversias en los acuerdos económicos internacionales, y proporcionará ejemplos de posibles conflictos resultantes de la superposición. También será observado el procedimiento internacional de elaboración de los tratados en el derecho económico internacional, en particular el uso de un lenguaje específico en la OMC y en los AII, así como las referencias en estos tratados y la jurisprudencia resultante de las disposiciones paralelas y las decisiones relacionadas con el comercio y/o la inversión. Por fin, serán discutidas las interacciones (tanto sustantivas como procesales) entre los AII y las disposiciones sobre solución de controversias de otros tratados, en particular el Acuerdo sobre la OMC. El artículo concluye que el efecto "spaghetti bowl" puede (por el momento) no ser tan perjudicial para la eficiencia como afirman algunos. Será discutida también la necesidad de mejorar los mecanismos institucionales para aumentar la coherencia, tanto en el ámbito de la inversión como en el comercio (OMC), aunque políticamente este reto no sea un logro fácil.

\section{DISPOSICIONES DE SOLUCIÓN DE CONTROVERSIAS SUPERPUESTAS EN LOS ACUERDOS ECONÓMICOS INTERNACIONALES}

OMC, los ACR y los AII

La coexistencia de la OMC, los ACR y los AII puede llevar a problemas legales en lo que respecta al uso y los resultados de sus respectivas disposiciones de solución de controver$\operatorname{sias}^{14}$. Especialmente a finales de 1990, el Entendimiento sobre Solución de Diferencias de la OMC (ESD) fue recibido como un importante logro para la resolución de disputas comerciales. El número de notificaciones de controversias y el número de grupos especiales e informes del Órgano de Apelación es bastante significativo, en comparación con las cifras generales de casos juzgados por los tribunales y cortes internacionales ${ }^{15}$.

virtud de un AII (Suiza - Uruguay). Véase News in Brief: Uruguay prepares defense against Philip Morris. Investment Treaty News, 16 diciembre 2010, Disponible en internet: IISD < http://www.iisd.org/ itn/2010/12/16/news-in-brief-2/>.

13 De Mestral, Armand. nafta Dispute Settlement: Creative Experiment or Confusion. En: Bartels, Lorand y Ortino, Frederico (eds.). Regional Trade Agreements and the WTO Legal System. Oxford: Oxford University Press, 2006, p. 359.

14 Véase también Hillman, JenNifER. Conflicts between dispute settlement mechanisms in regional trade agreements and the WTO. 42:2 Cornell Int'l LJ 193, 2009; PAuWELYN, JoOST y SALLES, Luiz Eduardo. Forum shopping before international tribunals. 42:1 Cornell Int'l LJ 77, 2009.

15 Según la OMC, el 1. ${ }^{\circ}$ de julio de 2014, 482 disputas fueron registradas bajo el sistema de la OMC. 123 casos dieron lugar a un informe del órgano hasta el $1 .^{\circ}$ de julio de 2014. Disponible en internet: OMC: $<$ http://www.wto.org/english/tratop_e/dispu_e/dispu_e.htm\#disputes $>$. Para más estadísticas, véase 
Al mismo tiempo, la OMC se omite en cuanto a la existencia de procedimientos de solución de diferencias específicas en otros acuerdos, y es necesario referirse al texto del ESD y, posiblemente, a las excepciones sobre los ACR que se encuentran en el Acuerdo General sobre Aranceles Aduaneros y Comercio (GATT, por su sigla en inglés) y el Acuerdo General sobre el Comercio de Servicios (AGCS) para abordar la cuestión ${ }^{16}$. Aunque el número de casos relacionados con los procedimientos de solución de diferencias comerciales de los ACR parece bastante pequeño ${ }^{17}$ hasta el presente momento, hay un número creciente de laudos arbitrales en virtud de los AII y los capítulos de inversión de $\operatorname{los} \mathrm{ACR}^{18}$. A menudo, no hay un análisis comparativo o integral con respecto a las reglas del comercio que figuran en los ACR o de la OMC, pero se observa una creciente superposición en cuanto a la interpretación de ciertos términos o el uso de los foros y procedimientos específicos (forum o treaty shopping) ${ }^{19}$.

\section{Situaciones típicas}

La disputa sobre la madera blanda (o disputas, dependiendo de la consideración de las distintas medidas contenciosas y su momento) entre los Estados Unidos y Canadá no solo ha dado lugar a diversas reclamaciones del TLCAN ${ }^{20}$, sino que también ha sido abordada por la OMC y, más recientemente, incluso por procedimientos tradicionales de arbitraje

Leitner, Kara y Lester, SimOn. WTo Dispute Settlement 1995 - 2013 - A Statistical Analysis. 17 (1): 191-201, 2014. Véase Understanding on Rules and Procedures Governing the Settlement of Disputes [DSU]. En: OMC, The Legal Texts: The Results of the Uruguay Round of Multilateral Trade Negotiations. Cambridge: Cambridge University Press, 1999. [WTO Agreements].

16 Véase DSU, GATT and General Agreement on Trade in Services (GATS). En: WTO Agreements, ibid. La decision más importante es Turkey - Restrictions on Imports of Textile and Clothing Products (1999), WT/DS34/AB/R (Appellate Body Report). Disponible en internet: WTO < http://www.wto.org/english/ tratop_e/dispu_e/34abr_e.pdf>. Una decisión más significativa es Brazil - Measures affecting Imports of Retreaded Tires (2007). WT/DS332/AB/R (Appellate Body report). Disponible en internet: WTO <http:// www.wto.org/english/tratop_e/dispu_e/cases_e/ds332_e.htm >. Para una propuesta de como resolver un possible conflicto, véase también OdEndAHL, KERSTIN. Prozessuale Wege zur Vermeidung von Jurisdiktionskonflikten im Völkerrecht - unter besonderer Berücksichtigung des WTO-Streitbeilegungsverfahrens. En: Lorandi, Franco y StaEHlin, Daniel (eds.). Innovatives Recht, ES Ivo Schwander (Zürich, Dike, 2011), 87-105.

17 Tal vez con la excepción de los TLC y el MERCOSUR. Véase, para estos últimos y los demás acuerdos en América Latina: Disponible en internet: UN IDATD < http://idatd.eclac.cl/controversias/index_en.jsp. > .

18 La CNUCED reportó 568 casos inversionista - Estado conocidos a finales de 2013. Véase CNUCED. Recent Developments in Investor - State Dispute Settlement. IIA Issues Note 1, 2014. Disponible en internet: UNCTAD < http://unctad.org/en/PublicationsLibrary/webdiaepcb2014d3_en.pdf>

19 Véase Marceau, Gabrielle y Wyatt, Julian. Dispute Settlement Regimes Intermingled: Regional Trade Agreements and the WTO. 1:1 Journal of International Dispute Settlement 67, 2010. Véase también BlysChaK, Paul Michael. Access and advantage expanded: Mobil Corporation v Venezuela and other recent arbitration awards on treaty shopping.4:1 J World Energy Law Bus 32-39, 2011.

20 Véase, sobre los varios procedimientos disponibles, Stout, TAYLOR G. NAFTA Dispute Settlement Procedures. International Judicial Monitor, 2011. Disponible en internet: International Judicial Monitor <http://www.judicialmonitor.org/archive_winter2011/spotlight.html> o QUAYAT, DAVID. The forest for the trees: a roadmap to Canada's litigation experience in lumber IV. 12:1 JIEL 115-151, 2009. 
bilateral fuera del ámbito del TLCAN y de la OMC. Es un ejemplo típico de la superposición que puede surgir en situaciones económicas complejas, en las obligaciones contraídas en virtud de varios tratados en diversas áreas del derecho económico internacional, en particular, el comercio y la inversión. La complejidad de la controversia pasó por varias fases y una gran cantidad de procedimientos. Podemos citar, por ejemplo, la llamada disputa sobre madera blanda III (1991-1996), que inició un procedimiento bajo el capítulo 19 del Acuerdo Estados Unidos - Canadá de Libre Comercio (UCFTA, el predecesor del TLCAN) y el Acuerdo sobre Subvenciones y Medidas Compensatorias del antiguo GATT de 1947 (antes de la entrada en vigor del Acuerdo sobre la OMC). Por fin, un acuerdo ad boc de 13 páginas entre Estados Unidos y Canadá decidió el caso. En una fase posterior de la controversia, referida regularmente como "madera blanda IV" (2001-2006), once paneles bajo el capítulo 19 del TLCAN (antidumping / derechos compensatorios) fueron constituidos, además de cuatro tribunales en virtud del capítulo 11 del TLCAN (Inversión) y seis grupos especiales de solución de controversias de la OMC. Aparte de esta gran cantidad de casos internacionales, debido a la construcción de las disposiciones del TLCAN, cinco casos de la Corte de Comercio Internacional de los Estados Unidos, dos casos de la Corte Distrital y tres peticiones del Tribunal de Apelaciones han sido presentados. Los casos más recientes de la disputa fueron objeto de dos sentencias arbitrales por la Corte de Arbitraje Internacional de Londres, en $2009^{21}$ y $2011^{22}$.

La disputa sobre edulcorantes o refrescos es otro caso que ejemplifica el uso de diversos procedimientos de solución de controversias en materia de inversión y comercio, que abordan cuestiones comerciales típicamente discutidas en la OMC y el TLCAN (capítulo 19), mientras otros aspectos fueron presentados por inversores privados ante tribunales arbitrales inversionista - Estado ${ }^{23}$. Este caso se refiere a una serie de medidas relacionadas con el comercio y la inversión adoptadas por México para proteger a sus productores de azúcar contra las importaciones de los Estados Unidos, y la producción local de jarabe de maíz rico en fructosa más barato que se utilizaría en la producción de refrescos. Esto resultó en la impugnación en la OMC de ciertos derechos antidumping de México por parte de los Estados Unidos ${ }^{24}$, mientras que México trató de llevar a los Estados Unidos al arbitraje de Estado a Estado bajo el capítulo 20 del TLCAN, en un esfuerzo por obligar a los Estados Unidos a permitir un mayor nivel de las importaciones

Véase United States of America v Canada, Decision, LCIA Case n. ${ }^{\circ}$ 91312, 27 septiembre 2009

22 United States of America v Canada, laudo arbitral, Corte de Arbitraje Internacional de Londres Case n. ${ }^{\circ} 81010,20$ y 28 enero 2011.

23 Véase DaveY, William J y SaPIR, André. The Soft Drinks Case: The WTO and Regional Agreements. 8:1 World Trade Review 5, 2009; HAMELMANN, SANDY. Internationale Jurisdiktionskonflikte und Vernetzungen transnationaler Rechtsregime: Die Entscheidungen des Panelyys und des Appellate Body der WTO in Sachen 'Mexico - Tax Measures on Soft Drinks and Other Beverages'. 58 Beiträge zum transnationalen Wirtschaftsrecht 1, 2006, y GUGLYA, LEONILA. The Interplay of International Dispute Resolution Mechanisms: the Softwood Lumber Controversy.2:1 J Int. Disp. Settlement 175-207, 2011.

24 Mexico - Anti-Dumping Investigation of High-Fructose Corn Syrup (HFCS) from the United States (Complaint by the United States), 2001. OMC Doc WT/DS132/AB/RW (Appellate Body Report). Disponible en internet: OMC < http://wto.org/english/tratop_e/dispu_e/cases_e/ds132_e.htm >. 
de azúcar mexicano libre de impuestos ${ }^{25}$. No obstante, México acusó a Estados Unidos de bloquear el nombramiento de un tribunal para conocer este reclamo bajo el TLCAN, y le pidió al Grupo Especial de la solución de diferencias de la OMC tener esto en cuenta y desestimar la reclamación ante la OMC en favor de los procedimientos del TLCAN, lo que fue rechazado por el Grupo Especial y el Órgano de Apelación de la OMC, ya que carecían del poder para hacerlo ${ }^{26}$. Asimismo, el 31 de diciembre de 2001, el Congreso mexicano aprobó un nuevo impuesto del 20 \% sobre los refrescos y jarabes endulzados con edulcorantes distintos del azúcar. Este impuesto dio origen a tres demandas separadas por inversionistas de los Estados Unidos, en virtud del capítulo 11 del TLCAN. Además, el impuesto resultó en procedimientos bajo la $\mathrm{OMC}^{27}$ y el TLCAN contra México iniciados por el gobierno de los Estados Unidos. México derogó el impuesto en el inicio de 2001, como parte del cumplimiento del fallo de la OMC. Sin embargo, los demandantes privados persistieron con su reclamo bajo el capítulo 11 del TLCAN, buscando daños y perjuicios por las pérdidas durante el período en que el impuesto estuvo en vigencia ${ }^{28}$.

\section{DIVERSIDAD DE TRATADOS EN MATERIA DE DERECHO INTERNACIONAL ECONÓMICO}

Tratados que regulan o prevén aspectos de las relaciones económicas bilaterales tienen una larga tradición. A finales de los siglos XVII y XIX, estos tratados, en general, eran instrumentos integrados que comprendían el comercio y el derecho de establecimiento entre las partes contratantes, y el trato de los nacionales de otra parte contratante. La forma más común era el Tratado de Amistad, Comercio y Navegación entre dos o más

Véase Mexico - Measures on Soft Drinks and Other Beverages (Complaint by the United States), 2005, OMC Doc WT/DS308/R (Informe del Panel). Disponible en internet: OMC <http://www.wto.org/english/ tratop_e/dispu_e/cases_e/ds308_e.htm> at para 4.92 [Mexico Soft Drinks].

26 Véase Mexico Soft Drinks, ibid; Mexico - Taxes Measures on Soft Drinks and Other Beverages (Complaint by the United States), 2006. OMC Doc WT/DS308/AB/R (Informe del Órgano de Apelación). Disponible en internet: OMC <http://www.wto.org/english/tratop_e/dispu_e/cases_e/ds308_e.htm >. [Mexico-Soft Drinks Appellate Body]. Véase PAUwELYN, Joost. Adding Sweeteners to Softwood Lumber: the WTONAFTA 'Spaghetti Bowl' is Cooking. 9:1 J Int'l Econ L 197, 2006. Véase también, sobre la cuestión de si el Grupo Especial y el Órgano de Apelación deberían haber tenido en cuenta las obligaciones de Brasil dentro del MERCOSUR, Brazil - Measures Affecting Imports of Retreaded Tyres (Complaint by the European Communities), 2007. OMC Doc WT/DS332/AB/R (Informe del Órgano de Apelación). Disponible en internet: OMC <http://www.wto.org/english/tratop_e/dispu_e/cases_e/ds332_e.htm>. Véase, sobre esto, Dias Varella, Marcelo y Freitas Filho, Roberto. The World Trade Organization as a Catalyst for Internal Divergences in Developing Countries. 4:9 Global Trade and Customs Journal 281-291, 2009, y MorosinI, Fabio. The MERCosur Trade and Environment Linkage Debate: The Disputes over Trade in Retreaded Tires.44:5 Journal of World Trade 1127-1144, 2010.

27 Mexico Soft Drinks, supra nota 18, y Mexico Soft Drinks Appellate Body, supra nota 19.

28 Véase, Archer Daniels Midland Company and Tate \& Lyle Ingredients Americas, Inc. v United Mexican States, Award, ICSID Case n. ${ }^{\circ}$ ARB (AF)/04/5, 21 noviembre 2007; Corn Products International, Inc. v United Mexican States, Decision on Responsibility, ICSID Case n. ${ }^{\circ}$ ARB (AF)/04/1, 15 enero 2008 ${ }_{i}$ Cargill, Inc. v United Mexican States, Award, ICSID Case n. ${ }^{\circ}$ ARB (AF)/05/2, 18 septiembre 2009. 
estados. Para los países sin litoral, estos tratados se denominaban Tratado de Amistad, Comercio y Establecimiento.

Después de la Segunda Guerra Mundial, falló el intento de crear un tratado multilateral en el contexto de la Conferencia de las Naciones Unidas sobre Comercio y Empleo. No solo era imposible establecer la Organización Internacional de Comercio (OIC) con un mandato amplio, sino que los intentos de codificar las reglas de inversión habían, incluso antes de la firma del Acta Final, llevado a resultados limitados. Esto se debió, en gran parte, a la ausencia de un consenso claro sobre el alcance y el papel de esas normas ${ }^{29}$.

Mientras que la creación de la OMC en 1994 dio lugar a un marco verdaderamente multilateral para el comercio y la resolución de disputas comerciales, el área de la inversión y las disputas sobre inversiones se mantiene al margen de este marco. Esto ha llevado a una dicotomía clásica en la enseñanza y en las negociaciones de los dos campos como "derecho internacional del comercio" y "derecho internacional de la inversión"30. Aunque se haya discutido la inclusión de normas sobre inversión en la OMC, la mayor superposición directa de los dos regímenes se relaciona con el Acuerdo sobre Medidas de Inversión Relacionadas con el Comercio (MIC) y el acceso al mercado y tratamiento de los proveedores extranjeros de servicios (modo 3 del AGCS) ${ }^{31}$.

La situación se ha vuelto aún más confusa, a medida que más $\mathrm{AII}^{32}$ incluyen procedimientos ambiciosos de solución de controversias inversionista - Estado que permiten que los inversores cuestionen las medidas del Estado receptor de una manera bastante completa, conduciendo a posibles superposiciones entre las medidas de inversión en virtud de un AII y medidas comerciales reguladas por la OMC. Esta tendencia se ha visto agravada por el creciente número de ACR negociados desde principios de los años noventa, que incluyen normas de comercio y de inversión ${ }^{33}$, normalmente acompañados de varios tipos de procedimientos de solución de diferencias ${ }^{34}$. En algunos casos, inclu-

29 Para más detalles, véase ZIEGLER, ANDREAS R. Multilateraler Investitionsschutz im Wirtschaftsrecht. En: EHLERS, DiRK y WolfFGANG, HANS-MiCHAEL (eds.). Rechtsfragen internationaler Investitionen. Frankfurt: Verlag Recht und Wirtschaft, 2009.

30 Véase QuRESHI, ASIF y Ziegler, ANDrEas R. International Economic Law. 3 ed. London: Thomson Sweet \& Maxwell, 2011.

31 Véase MIC y AGCS en los acuerdos de la OMC, supra nota 12. Véase AdLunG, Rudolf y MolinUEVO, MARTín. Bilateralism in Services Trade: Is There Fire Behind the (BIT) Smoke. 11:2 JIEL 365, 2008. Disponible en internet: JIEL http://jiel.oxfordjournals.org/content/11/2/365.abstract.

32 Según la CNUCED, a finales de 2013 había 2.902 TBI, otros 334 acuerdos internacionales con disposiciones relativas a las inversiones. Véase CNUCED, World Investment Report 2014: Investing in the SDGs: an action plan (New York and Geneva: UNCTAD, 2014). p .114.

33 Véase WTO RTA Gateway, Disponible en internet: WTO<http://www.wto.org/english/tratop_e/region_e/ region_e.htm>. A 15 de junio de 2014, se habían notificado al GATT/OMC unos 585 ACR (si se cuentan por separado bienes y servicios), de los cuales 224 tratados fueron notificados en virtud del artículo XXIV del GATT de 1947 o del GATT de 1994; 34, en virtud de la Cláusula de Habilitación, y 119, en virtud del artículo V del AGCS; en esa misma fecha, 379 acuerdos estaban en vigor.

34 Véase PAUWELYN. Adding Sweeteners. supra nota 19. 
so los ACR más antiguos han sido enmendados para incluir mecanismos de solución de controversias no previstos en los acuerdos originales ${ }^{35}$.

Desde una perspectiva jurídica, el artículo XXIV del GATT ${ }^{36}$ y el artículo V del AGCS ${ }^{37}$ -que permiten a los miembros de la OMC ser parte de estos acuerdos- han sido objeto de largas negociaciones y tensiones dentro de la OMC. La mayoría de los estados argumenta que solo ingresan en tratados que son plenamente compatibles con sus obligaciones en la $\mathrm{OMC}^{38}$, y a menudo esto ha sido cuestionado. Además, desde hace varios años la coexistencia entre la OMC y los ACR ha sido descrita como un "spagbetti bowl", donde las disciplinas y compromisos de la OMC solo se aplican a un grupo cada vez más restricto de miembros, mientras muchos se basan en normas preferenciales, perjudicando la transparencia y aumentando las distorsiones en los flujos comerciales internacionales ${ }^{39}$.

\section{AII Y MECANISMOS DE SOLUCIÓN DE CONTROVERSIAS EN OTROS TRATADOS}

\section{La superposición sustantiva}

La posibilidad de aplicar diversos mecanismos de solución de diferencias de los distintos acuerdos a la misma situación deriva de las provisiones de algunos ACR que afirman la existencia de derechos y obligaciones existentes con arreglo al Acuerdo de la OMC o de otro tratado. Por ejemplo, el artículo 1.2 del Acuerdo de Promoción Comercial Perú Estados Unidos confirma las obligaciones de las partes en virtud de otro acuerdo y, en particular, de la OMC ("Las Partes confirman los derechos y obligaciones existentes entre ellas conforme al Acuerdo sobre la OMC y otros acuerdos de los que sean parte"). Esto se repite a lo largo del tratado, por ejemplo en relación con el Acuerdo sobre Obstáculos

35 Véase el acuerdo entre Túnez y la Comunidad Europea (ahora Unión Europea): EC, Council Decision 2010/91/EC of 10 November 2009 on the conclusion of an Agreement in the form of a Protocol between the European Community and the Republic of Tunisia establishing a dispute settlement mechanism applicable to disputes under the trade provisions of the Euro-Mediterranean Agreement establishing an Association between the European Communities and their Member States, of the one part, and the Republic of Tunisia, of the other part, [2010] OJ L 40/75 [EC-Tunisia Protocol Agreement]; véase, sobre esto, Ziegler, ANDreas R. Droit international économique de la Suisse - Une introduction. Berne: Stämpfli, 2010.

36 Véase GATT en los acuerdos de la OMC, supra nota 12.

37 Véase AGCS en los acuerdos de la OMC, supra nota 12.

38 Esto incluso figura como una declaración visible en el texto (a menudo el preámbulo) de la mayoría de los ACR. Disposiciones que confirman obligaciones de la OMC de las partes son muy comunes en los acuerdos comerciales regionales, en lo que respecta a las diversas áreas cubiertas.

39 El Comité de la OMC, el 4 de octubre de 2010, aprobó una propuesta de Brasil, China, India y los Estados Unidos para establecer un mecanismo de transparencia para los acuerdos comerciales preferenciales adoptados por el Consejo General, en su sesión del 14 de diciembre de 2010. Véase Consejo General de la OMC, acta de la reunión (celebrada el 14 de diciembre de 2010), OMC Doc WT/COMTD/71. Disponible en internet: WTO <http://www.wto.org/english/thewto_e/gcounc_e/meet_dec10_e.htm>; Consejo General de la OMC, Minutes of Meeting (celebrada el 14 de diciembre de 2010), OMC Doc WT/L/806. Disponible en internet: WTO < http://www.wto.org/english/news_e/news10_e/summary_gc_dec10_e. htm>. 
Técnicos al Comercio (OTC) ${ }^{40}$. Lo mismo ocurre en otros acuerdos, como los celebrados por la Asociación Europea de Libre Comercio (AELC) ${ }^{41}$.

En ciertos casos, las partes en los ACR repiten de forma explícita las obligaciones ya existentes (de la OMC) o se refieren a ellas. Esto puede conducir a una llamada incorporación de esas normas y, normalmente, hace que su violación también sea una violación del acuerdo en el que se confirman. Esta es una práctica bien conocida (ya en relación con el GATT de 1947) bajo los acuerdos entre el TLCAN, la UE y la AELC con terceros Estados. Un ejemplo es el artículo 7.3.2 del Acuerdo de Promoción Comercial Perú - Estados Unidos que ha incorporado explícitamente el trabajo existente con respecto a las normas del Acuerdo OTC realizado dentro de la OMC.

Si esto sucede en un AIl o en el capítulo de inversiones de un ACR, puede tener el efecto adicional de incorporar disciplinas de la OMC o de otros acuerdos en el acuerdo bilateral o regional, y de estar sujeto al arbitraje inversionista - Estado, que es típico de los dos acuerdos de inversión y los capítulos de inversión. El artículo 10.7.5 del Acuerdo de Promoción Comercial Perú - Estados Unidos ejemplifica bien el caso. En el contexto de la definición de una expropiación en el capítulo de inversiones del acuerdo (capítulo 10), el acuerdo incorpora normas de la OMC. Con el fin de evaluar la legalidad de una licencia obligatoria, las partes se refieren a las normas del Acuerdo sobre los Aspectos de los Derechos de Propiedad Intelectual relacionados con el Comercio (ADPIC). Esta práctica existe también en otros AII y ACR ${ }^{42}$. Algunos AII incluso excluyen explícitamente

40 Acuerdo de Promoción Comercial Perú-Estados Unidos, 12 de abril de 2006. Disponible en internet: Gobierno del Perú <http://www.acuerdoscomerciales.gob.pe/images/stories/eeuu/espanol/Disposiciones_\%20Iniciales.pdf> [EE.Uu. - Peru TLC].

41 En el Acuerdo de Libre Comercio entre los Estados de la AELC y Perú de 24 de junio de 2010, el preámbulo incluye las siguientes declaraciones: "Construyendo sobre la base de sus respectivos derechos y obligaciones bajo el Acuerdo de Marrakech que establece la Organización Mundial de Comercio (en adelante el "Acuerdo de la OMC") y los demás acuerdos negociados bajo dicho marco y los demás instrumentos multilaterales y bilaterales de cooperación" y en el Artículo 1.4. (Relación con Otros Acuerdos Internacionales) se lee: "Las disposiciones de este Acuerdo serán aplicables sin perjuicio de los derechos y obligaciones de las Partes bajo el Acuerdo de la OMC y los otros acuerdos negociados con arreglo al mismo de los cuales sean parte y cualquier otro acuerdo internacional de los cuales sean parte". Véase Acuerdo de Libre Comercio entre los Estados de la AELC y Perú, 24 junio 2010. Disponible en internet: Gobierno del Perú <http://www.acuerdoscomerciales.gob.pe/images/stories/efta/espanol/Acuerdo_Principal.pdf $>$ at III, art 1.4 .

42 Véase Acuerdo sobre los Aspectos de dos Derechos de Propiedad Intelectual relacionados con el Comercio (Acuerdo sobre los ADPIC) en los Acuerdos de la OMC, supra nota 12. Lo mismo puede ser visto en Acuerdo entre la República de Colombia y la Confederación Suiza sobre la Promoción y Protección Recíproca de las Inversiones, 17 mayo 2006. Disponible en internet: <http://www.seco.admin.ch/themen/00513/00594/04638/index.html?lang=en> 15: (1) (1It is understood that the said Article is without prejudice to the issuance of compulsory licenses granted in relation to intellectual property rights or other measures taken in accordance with the WTO Agreement on Trade-Related Aspects of Intellectual Property Rights". Véase detalladamente sobre este asunto: GiBSON, CHRISTOPHER. A Look at the Compulsory License in Investment Arbitration: The Case of Indirect Expropriation. 25 Am U Int'l L Rev 357, 2010. 
la posibilidad de impugnar la compatibilidad con la OMC de las licencias obligatorias, con arreglo a sus disposiciones sobre solución de controversias inversionista - Estado ${ }^{43}$.

Del mismo modo, la incorporación de las Medidas en Materia de Inversiones Relacionadas con el Comercio (MIC) de la OMC en los AII o capítulos de inversión de los ACR es muy común, como, por ejemplo, es el caso del artículo 96 del acuerdo de libre comercio entre Suiza y Japón de $2009^{44}$.

El acuerdo trilateral de libre comercio entre la ASEAN, Australia y Nueva Zelanda también contiene una disposición similar ${ }^{45}$. En el artículo 5 del capítulo 11 (Inversión), el acuerdo incorpora las obligaciones existentes de la OMC bajo el acuerdo MIC. Este artículo, sin embargo, excluye el procedimiento de solución de controversias del capítulo de inversiones, posiblemente para evitar la interpretación del Derecho de la OMC por parte de los tribunales arbitrales inversionista - Estado ${ }^{46}$. No obstante, esta exclusión del mecanismo de solución de controversias no existe en otros acuerdos, como el ALC entre Japón y Suiza citado anteriormente, que también incorpora las provisiones del acuerdo MIC. Por tanto, el último caso puede resultar en un procedimiento de solución de controversias inversionista - Estado en el que el tribunal arbitral podría tener que interpretar las provisiones del acuerdo MIC.

La tendencia generalizada de incorporar las excepciones de la OMC en capítulos de inversión de los ACR es particularmente importante ${ }^{47}$. Un ejemplo típico (pero que se refiere al comercio de servicios) es el artículo 5.8 del TLC entre la AELC y Perú: "la excepción en el subpárrafo (d) del Artículo XIV del AGCS, se incorpora y forma parte del presente Capítulo, mutatis mutandis" ${ }^{\prime \prime 8}$. Otro ejemplo de una cláusula de excepción basada en la

Véase TIB Modelo de la República de Colombia, 2007. Disponible en internet: Italaw <http://italaw.com/ documents/inv_model_bit_colombia.pdf> art 6.7. ("The Contracting Parties confirm that issuance of compulsory licenses granted in accordance with the TRIPS Agreement of the WTO, may not be challenged under the provisions set out in this Article").

"Article 96 Prohibition of Performance Requirements: For the purposes of this Chapter, the Annex to the Agreement on Trade-Related Investment Measures in Annex 1A to the WTO Agreement is hereby incorporated into and made part of this Agreement, mutatis mutandis". Agreement on Free Trade and Economic Partnership between Switzerland and Japan, 19 febrero 2009. Disponible en internet: Seco $<$ http://www.seco.admin.ch/themen/00513/02655/02731/02970/index.html?lang=en> art 96 [Switzerland- Japan FTA].

45 ASEAN - Australia - New Zealand Free Trade Agreement, 27 febrero 2009. Disponible en internet: ASEAN $<$ http://www.asean.fta.govt.nz/chapter-11-investment/>.

46 Ibid ("Claim by an Investor of a Party: If an investment dispute has not been resolved within 180 days of the receipt by a disputing Party of a request for consultations, the disputing investor may, subject to this Article, submit to conciliation or arbitration a claim: that the disputing Party has breached an obligation arising under Article 4 (National Treatment), Article 6 (Treatment of Investment), Article 7 (Compensation for Losses), Article 8 (Transfers), and Article 9 (Expropriation and Compensation)..." at art 20).

47 Véase Newcombe, Andrew. Cap. 16. En: De Mestral, Armand y Lévesque, Céline (eds.). Improving International Investment Agreements. London: Routledge, 2012.

48 Acuerdo de Libre Comercio entre la República del Perú y los Estados AELC, 14 julio 2010. Disponible en internet: http://www.sice.oas.org/Trade/PER_EFTA/Text_s.asp\#a58 
OMC en un capítulo de inversiones de un ACR se puede encontrar en el artículo 95 del TLC entre Suiza y Japón ${ }^{49}$.

Además de la referencia a la incorporación de las obligaciones existentes de otros acuerdos en los AII, la superposición sustancial puede resultar del ámbito de aplicación de algunas nociones vagas con respecto al tratamiento y la protección utilizadas generalmente en los AII. Varios autores han analizado la posibilidad de que los inversores recurriesen a normas típicas de tratamiento de los AII para cuestionar posibles violaciones de la OMC (o de otros acuerdos) por parte del Estado receptor ${ }^{50}$. Esta cuestión también fue abordada por algunos tribunales ${ }^{51}$. Algunas consideraciones sobre el recurso a la superposición sustancial para provocar una violación de otra obligación del tratado dentro del alcance del mecanismo de solución de diferencias de la IIA son descritas abajo ${ }^{52}$ :

- Los casos anteriores consideraban de gran importancia la posibilidad de interpretar la obligación de "trato justo y equitativo", para imponer al Estado receptor el cumplimiento de todas sus obligaciones en virtud del derecho internacional, incluidas las obligaciones de tratados externos al AII. Los tribunales arbitrales, hasta ahora, han evitado responder claramente a esta pregunta, y se basan en una interpretación autónoma de las normas de los tratados de inversión ${ }^{53}$.

- La doctrina ha identificado que las "cláusulas paraguas", que convierten cualquier otra obligación legal impuesta por el Estado en una posible violación del AII, pueden permitir que los inversores presenten una demanda del AIl basada en la violación de otro tratado ${ }^{54}$.

- Un trato discriminatorio de las importaciones puede fácilmente ser considerado una violación del trato nacional y/o las normas de la nación más favorecida previstas en la mayoría de los AII, si se puede demostrar que este trato discriminatorio afecta a la inversión extranjera y el inversor extranjero ${ }^{55}$.

49 Article 95 General and Security Exceptions. 1.In respect of the making of investments, Articles XIV and XIVbis of the GATS, which are hereby incorporated into and made part of this Agreement, mutatis mutandis, shall apply. 2.Paragraph 1 of Article XIvbis of the GATS shall also apply, mutatis mutandis, to investments made. This Article shall not apply to paragraph 1 of Article 86 [General Treatment], and Articles 91 [Expropriation] and 92 [Treatment in Case of Strife]". Switzerland- Japan FTA, supra nota 41.

50 Véase VerhoOsell, GaETAN. The Use of Investor-State Arbitration under Bilateral Investment Treaties to Seek Relief for Breaches of WTO Law. 6:2 JIEL 493, 2003.

51 Véase Grand River Enterprises et al. v United States of America, laudo arbitral, UNCITRAL, 12 enero 2011, [Grand River].

52 Lo contrario también es posible. En una decisión de la Corte Europea de Derechos Humanos, la no ejecución de un laudo arbitral se considera una violación del Protocolo Adicional a la Convención Europea de Derechos Humanos. Véase Kin-Stib and Majkíc v. Serbia, n. ${ }^{\circ}$ 12312/05, 20 abril 2010.

53 Véase Owen Verrill Jr, Charles. Are wTo Violations Also Contrary to the Fair and Equitable Treatment Obligations in Investor Protection Agreements. 11:2 ILSA Journal of International and Comparative Law $287,2005$.

54 Véase VerHoOSELL, Gaetan supra nota 47

55 Véase también KURTZ, JÜrGEN. National Treatment, Foreign Investment and Regulatory Autonomy: The Search for Protectionism or Something More? En: P KAHN y T WÄLDE (eds.). New Aspects of International Investment Law. Leiden: Martinus Nijhoff, 2007. Además, los diferentes conceptos de la 
- Cualquier violación de los derechos de propiedad intelectual con arreglo al acuerdo sobre ADPIC puede ser fácilmente interpretada como un tratamiento no justo y equitativo, conforme a lo solicitado en la mayoría de los AII, o incluso constituir una expropiación a los derechos de propiedad intelectual cubiertos por el IIA (generalmente este es el caso). Por ejemplo, varios foros discuten los nuevos requisitos de etiquetado para los cigarrillos. La obligación de vender cigarrillos en paquetes genéricos estándar, particularmente, es muy controvertida y ha dado lugar a casos iniciados por el gigante del tabaco Philip Morris International (PMI) contra Uruguay y Australia en virtud de un AII, por un lado ${ }^{56}$, y debates en la OMC en relación con el acuerdo sobre ADPIC, por otro lado ${ }^{57}$.

- Muchos AII se refieren al derecho internacional (general) con relación al trato de los inversores extranjeros y la inversión, sin delimitar las áreas o fuentes de derecho específicos. Generalmente, los tratados exigen un trato no menos favorable al "que exige el derecho internacional", como la mayoría de los AII negociados por los Estados Unidos ${ }^{58}$. Esto podría, por supuesto, ser utilizado para argumentar que la violación de una obligación en virtud del derecho internacional se considerará una violación del AIl regida por la solución de controversias disponible solo para las violaciones de los AII. Este tema fue abordado explícitamente por las partes del TLCAN en una interpretación de la Comisión de Libre Comercio del TLCAN de 31 de julio de 2001: "Una resolución en el sentido de que se baya violado otra disposición del TLCAN o de un acuerdo internacional distinto no establece que se baya violado el artículo 1105(1)" y el artículo 1105 "no requiere un trato adicional al requerido por el nivel mínimo de trato a los extranjeros propio del derecho internacional consuetudinario, o que vaya más allá de éste"59.

discriminación bajo la OMC y los AII pueden ser relevantes. Sobre este asunto, véase DIMASCIO, NICHOLAS y Pauwelyn, Joost. Nondiscrimination in Trade and Investment Treaties: Worlds Apart or Two Sides of the Same Coin? 102:1 AJlL 48, 2008.

56 En un procedimiento de solución de controversias inversionista - Estado iniciado en 2010, el gigante del tabaco Philip Morris International (PMI) comenzó un arbitraje ante el CIADI contra las nuevas reglas en Uruguay que requieren que el $80 \%$ de la superficie del paquete de cigarrillos contenga alertas gráficas de los peligros asociados con fumar. Véase FTR Holding S.A., Philip Morris Products S.A. and Abal Hermanos S.A. v Oriental Republic of Uruguay, ICSID Case n. ${ }^{\circ}$ ARB/10/7, 26 marzo 2010. Disponible en internet: Italaw <http://italaw.com/chronological_list_if_content.htm>. Phillip Morris también presentó una demanda similar contra Australia. El procedimiento de arbitraje fue formalmente instaurado en 2012, bajo el artículo 10 del acuerdo de protección y promoción de inversiones entre Hong Kong y Australia, de 1993. Véase Philip Morris Asia Limited (Hong Kong) v. The Commonwealth of Australia, PCA Case n. ${ }^{\circ} 2012-12,7$ junio 2012. Disponible en internet: http://www.pca-cpa.org/showpage.asp?pag_id $=1494$.

57 Un proyecto de ley australiano que requeriría que los cigarrillos $-\mathrm{y}$, finalmente, otros productos de tabaCO- sean vendidos en paquetes genéricos estándar fue objeto de escrutinio en la OMC en la reunión del Consejo de la Propiedad Intelectual (ADPIC) de 7 de junio 2011. Algunos miembros dijeron que podría violar los derechos de marca, mientras que otros la defendieron como una medida legítima para proteger la salud pública. Véase WTO, Members debate cigarette plain- packaging's impact on trademark rights, 7 junio 2011. Disponible en internet: WTO <http://www.wto.org/english/news_e/news11_e/trip_07jun11_e. htm>.

58 Véase TIB EE.UU. - Egipto (1986) en art II(4); TIB EE.Uu. - Argentina (1991) en art. II(2).

59 TLCAN Comisión del Libre Comercio. Notas interpretativas de ciertas disposiciones del Capítulo 11, 31 julio 2001. Disponible en internet: OEA: http://www.sice.oas.org/tpd/nafta/Commission/CH1 1 understanding_s. asp 
- En conclusión, existe un gran potencial de superposición de estas áreas del sistema de la OMC donde hay reglas adecuadas sobre inversiones, en particular-como fue mencionado anteriormente- en el modo 3 del suministro de servicios en el AGCS ${ }^{60}$.

Hasta el momento, los árbitros de los procedimientos inversionistas - Estado se han negado a permitir la importación de otras obligaciones derivadas de otros tratados a los AII. Parecen evitar, en la medida de lo posible, una situación en la que tienen que interpretar otros tratados (como los tratados de derechos humanos, los ACR o la OMC). Un posible argumento a favor de este enfoque es que la importación de otros tratados ampliaría la protección disponible y podría sobrecargar los AII, así como el equilibrio que se acordó en la negociación del AII. Un ejemplo es el laudo arbitral del caso Grand River vs. Estados Unidos, una disputa presentada por un inversionista canadiense en virtud del capítulo 11 del TLCAN ${ }^{61}$. Los árbitros se negaron a considerar la posibilidad de que un incumplimiento de obligaciones exteriores al TLCAN pudiese dar lugar a un incumplimiento de las obligaciones de trato garantizadas bajo el TLCAN. Para llegar a esta decisión, los árbitros han expresamente diferido de la nota interpretativa de 2001. El tribunal declaró que no "importaría" otras normas de derecho internacional al TLCAN, tampoco permitiría la alteración de la interpretación establecida a través de los procesos de interpretación normal de la Convención de Viena ${ }^{62}$. Algunos autores han afirmado que los árbitros deberían estar más abiertos a este tipo de argumentos, en virtud del artículo 31.3.c de la Convención de Viena, que establece que las interpretaciones deberán tener en cuenta "toda forma pertinente de derecho internacional aplicable en las relaciones entre las partes"63. Algunos autores afirman que el artículo 31.3.c no funciona como un mecanismo de "importación" de normas externas de un tratado determinado, como el TLCAN, pero permite que los demandantes soliciten el incumplimiento de obligaciones externas. También permite que otras normas pertinentes del derecho internacional informen la interpretación de un tribunal de arbitraje de un determinado tratado de inversión u obligación resultante de un acuerdo comercial ${ }^{64}$.

\section{Superposición de procedimientos}

Tradicionalmente, los ACR no prevén la posibilidad de superposición con el GATT de 1947. Además, incluso después de la entrada en vigor de la OMC como su sucesora, los

60 Esta discusión no será desarrollada en este capítulo.

61 Grand River, supra nota 48.

62 Ibid, par. 71.

63 Véase Convención de Viena sobre el derecho de los tratados, 23 mayo 1969, 1155 UNTS 331, 8 ILM.679 (entrada en vigor, 27 de enero de 1980).

64 Véase Peterson, Luke Eric. Analysis: Tribunal in Grand River v. U.S.A. arbitration declines to import non-investment law obligations into NAFTA; role of other "relevant" legal obligations in treaty interpretation under Vienna Convention is not discussed, 2006. Disponible en internet: IA Reporter $<$ http://www. iareporter.com/articles/20110306_3>. 
ACR, a menudo, no mencionan el caso o contienen cláusulas ambiguas a este respecto. Con relación a otros mecanismos de solución de diferencias, los ACR normalmente no contienen normas específicas. No obstante, un enfoque integral puede encontrarse en algunos acuerdos comerciales ${ }^{65}$. El nuevo acuerdo entre Túnez y la Unión Europea prevé la posibilidad del recurso a la solución de diferencias en el marco del acuerdo o de la $\mathrm{OMC}$, pero prohíbe la instauración del mecanismo si la materia ya es objeto de una disputa en el marco de la OMC o del tratado ${ }^{66}$.

Especialmente con respecto a las disputas comerciales y los Estados involucrados en estas, una serie de potenciales fricciones han sido identificadas, particularmente con relación a la OMC. En primer lugar, el demandado puede intentar desestimar una reclamación de la OMC mediante la invocación de una reclamación existente basada en una obligación en virtud de otro tratado ${ }^{67}$. Este argumento es inválido para los procedi-

65 Véase también, para Mercosur: Morales de Paula, Guilherme. A cláusula de eleição de foro do Protocolo de Olivos e seus efeitos contraproducentes para o Mercosul. 6:1 Revista Escuela Direito Pelotas. $167,2005$.

66 Véase EC- Tunisia Protocol Agreement, supra nota 32.

"Article 20 Relation with WTO obligations

1. Recourse to the dispute settlement provisions of this Protocol shall be without prejudice to any action in the WTO framework, including dispute settlement action.

2. However, where a Party has, with regard to a particular measure, instituted a dispute settlement proceeding, either under this Protocol or under the WTO Agreement, it may not institute a dispute settlement proceeding regarding the same measure in the other forum until the first proceeding has ended. In addition, a Party shall not seek redress for the breach of an obligation which is identical under the Association Agreement and under the WTO Agreement in the two forums. In such case, once a dispute settlement proceeding has been initiated, the Party shall not bring a claim seeking redress for the breach of the identical obligation under the other agreement to the other forum, unless the forum selected fails for procedural or jurisdictional reasons to make findings on that claim.

3. For the purposes of paragraph 2:

- dispute settlement proceedings under the WTO Agreement are deemed to be initiated by a Party's request for the establishment of a panel under Article 6 of the Understanding on Rules and Procedures Governing the Settlement of Disputes of the WTO (DSU) and are deemed to be ended when the Dispute Settlement Body adopts the panel's report, and the Appellate Body's report as the case may be, under Articles 16 and 17(14) of the DSU,

- dispute settlement proceedings under this Protocol are deemed to be initiated by a Party's request for the establishment of an arbitration panel under Article 5(1) and are deemed to be ended when the arbitration panel notifies its ruling to the Parties and to the subcommittee on industry, trade and services under Article 8.

4. Nothing in this Protocol shall preclude a Party from implementing the suspension of obligations authorised by the Dispute Settlement Body of the WTO. The WTO Agreement shall not be invoked to preclude a Party from suspending its obligations under this Protocol".

67 Véase Argentina - Definitive Anti Dumping Duties on Poultry WTO Doc WT/DS241/R (Panel Report), 2003. Disponible en internet: WTO <http://www.wto.org/english/traptop_e/dispu_e/cases_e/ds241_e. htm>- (Argentina solicitó al panel de la OMC para desestimar la demanda presentada por Brasil a la luz de la decisión anterior del panel del MERCOSUR o, en su defecto, para reconocer esta decisión como vinculante. El panel ha rechazado la demanda, ya que no podía considerar los procedimientos del MERCOSUR). Véase también HeNCKELS, CAROLINE. Overcoming Jurisdictional Isolationism at the WTO-FTA Nexus: A Potential Approach for the WTO. 19:3 EJIL 571, 2008 (Henckels sostiene que en el interés 
mientos entre inversionistas y Estados, puesto que el Estado de origen del inversionista no está directamente involucrado en el procedimiento arbitral en el marco del AII. En segundo lugar, un Estado demandado puede justificar que su conducta es incompatible con un tratado, al referirse a la conducta inconsistente del reclamante con otro tratado, como en el caso México - Impuestos sobre los refrescos (DS308), donde México justificó su impuesto (discriminatorio) a través de la necesidad de imposición del impuesto para asegurar el cumplimiento por los Estados Unidos de su obligación de establecer un panel del TLCAN para resolver la disputa del azúcar ${ }^{68}$.

Con relación a los capítulos de inversión en los ACR o AII, algunos tratados contienen la obligación de elegir un foro, pero, tradicionalmente, esta cláusula se refiere a la elección entre los procedimientos de arbitraje internacional de inversión y los tribunales nacionales (elección de foro, dilema) ${ }^{69}$. El problema con respecto a la elección de dicho foro radica en el hecho mismo de que, en virtud de los acuerdos comerciales (incluyendo la OMC), no hay posibilidad de que los inversores privados puedan utilizarlos y, por tanto, normalmente solo disponen de procedimientos de solución de controversias inversionista - Estado. Para excluir el recurso a tales procedimientos, en el caso en que el Estado de origen haya estado involucrado en la cuestión en los procedimientos relacionados con el comercio, parece exagerado y posiblemente incompatible con el espíritu de solución de controversias inversionista - Estado. A menudo, la intención de los Estados contratantes es permitir la defensa de los derechos de los inversores sin depender de la protección diplomática.

Las disposiciones sobre inversiones y solución de controversias en el MERCOSUR

La promoción y protección de las inversiones extranjeras en el marco del MERCOSUR fue abordada inicialmente por la Decisión 01/92 del Consejo del Mercado Común del MERCOSUR, que aprobó el Cronograma de Las Leñas. El Cronograma estableció las medidas iniciales que se adoptarían para alcanzar los objetivos principales del Tratado de Asunción de 1991, la creación de un mercado común con libre circulación de los factores de

de la administración efectiva de la justicia, el órgano judicial de la OMC debería utilizar su poder inherente de trato paritario para declinar el ejercicio de su competencia, a fin de que la disputa pueda ser resuelta por un tribunal de un TLC cuando una controversia está inextricablemente relacionada con una disputa en virtud de un TLC y, consecuentemente, el ejercicio de la jurisdicción no sería razonable en dichas circunstancias. Otro caso similar envuelve a la Unión Europea, que argumenta la imposibilidad de demanda por parte de los EE.UU. en el marco del Acuerdo SMC de la OMC, debido a la existencia de los acuerdos bilaterales de 1979 y 1992 entre la UE y los EE.UU. (CE - Medidas que afectan al comercio de grandes aeronaves civiles (2011), OMC Doc. WT/DS316/AB/R). Véase también México - Impuestos sobre los refrescos (DS308), supra nota 18 (México pidió al panel de la OMC para desestimar la demanda a favor de la disputa en el marco del TLCAN Capítulo 20 sobre las restricciones estadounidenses a las importaciones de azúcar: tanto el panel establecido como el Órgano de Apelación constataron que no había ningún poder en la ley aplicable que permitiese declinar su competencia).

68 Véase Mexico Soft Drink, supra nota 18 y texto correspondiente.

69 Véase QURESHI y ZIEGLER, supra nota 23, 523. 
producción, el establecimiento de un arancel externo común y la coordinación de las políticas macroeconómicas entre los Estados miembros ${ }^{70}$. La decisión determinaba al Grupo Mercado Común la producción de un acuerdo marco para promover y proteger la inversión extra e intrarregional ${ }^{71}$. Posteriormente, dos proyectos de tratados fueron aprobados y adoptados por el Consejo del Mercado Común, así como dos protocolos, a saber, el Protocolo de Colonia para la Promoción y Protección Recíproca de Inversiones en el MERCOSUR, firmado en Colonia de Sacramento el 17 de enero de 1994 (Protocolo de Colonia), y el Protocolo de Promoción y Protección de Inversiones provenientes de Estados Partes no pertenecientes al Mercosur, firmado en Buenos Aires el 5 de agosto de 1994 (Protocolo de Buenos Aires).

Ambos protocolos buscaban promover las inversiones, determinando su ámbito de aplicación, y ofreciendo disposiciones sobre el tratamiento y la solución de controversias que garantizaban a los inversores una amplia gama de derechos e instrumentos para proteger sus intereses. Aunque menos completo, el ámbito de aplicación y la protección proporcionada a los inversores en estos protocolos han sido comparados con los previstos en el Acuerdo de Libre Comercio de América del Norte ${ }^{72}$. Sin embargo, los Protocolos de Colonia y Buenos Aires no fueron ratificados por todos los Estados miembros y, por consiguiente, nunca entraron en vigor.

Cabe resaltar que los Protocolos de Colonia y Buenos Aires fueron redactados a principios de la década de 1990, cuando los países del MERCOSUR buscaban atraer inversiones extranjeras, de manera que se mantuviera un nivel suficiente de capital para financiar su desarrollo económico. El panorama económico actual es bastante distinto. Paralelamente a esta creciente fuerza económica, se ha producido una importante disminución de los ideales neoliberales básicos en América Latina, y muchos gobiernos revalúan los beneficios de la IED para sus economías. Como consecuencia de este desarrollo, no hay duda de que los Protocolos, en particular el Protocolo de Buenos Aires, parecen ser obsoletos, ya que ofrecen principalmente protección a los inversores de los países desarrollados, donde las empresas del MERCOSUR no tienen inversiones importantes ${ }^{73}$.

En 2010, el Consejo del Mercado Común del MERCosur aprobó la Decisión 30/10, que establece en su anexo las directrices para la creación de un Acuerdo de Inversiones en el Mercosur. Aparentemente, estas directrices parecen estar basadas en el Protocolo de Montevideo sobre el Comercio de Servicios, en lugar de las normas relativas a inversiones internacionales, como los TBI y los capítulos sobre inversiones de los acuerdos comerciales regionales. Esta estrategia puede perjudicar la promoción y protección de las inversiones

70 Tratado de Asunción, artículo 1. ${ }^{\circ}$, marzo 26, 1991.

71 Véase CMC Dec. 01/92, Anexo, Subgrupo de Trabajo n. ${ }^{\circ}$ 4, Políticas Monetaria y Fiscal relacionadas con el Comercio, párrafo 5.

72 Para más detalles, véase PiñEIRO, M. y Villarreal, F. Foreign Investment in Agriculture in MERCOSUR Member Countries, (julio de 2012). TKN Informe en IIDS.

73 Véase Mata Diz, Jamile Bergamaschine. The Protection of Foreign Direct Investment in Mercosur. En: Franca Filho, Marcílio Toscano, Lixinski, Lucas y Olmos Giupponi, María Belén (eds.). The Law of Mercosur. Oxford: Hart Publishing, 2010, p. 290. 
en MERCOSUR, por varias razones. El argumento principal es que la protección contenida en la mayoría de los tratados de inversión regionales, como el requisito de trato justo y equitativo, no está prevista en el acuerdo del MERCOSUR. Además, las directrices solo prevén para el acuerdo un mecanismo de solución de controversias Estado - Estado, una clara diferencia con el sistema previsto en los Protocolos de Colonia y Buenos Aires, que permitía el arbitraje entre inversores y $\operatorname{Estados}^{74}$. Cabe observar que las directrices no hacen ninguna referencia a las conexiones entre desarrollo sostenible e inversiones. La propuesta de un acuerdo de inversión con base en las directrices aún no se ha adoptado ni presentado y, consecuentemente, el MERCOSUR seguirá careciendo de la normativa para la promoción y protección de inversiones. En ausencia de un régimen de inversiones en el bloque, las reglas de inversión se encuentran en los TBI y en la legislación nacional.

En materia de solución de diferencias, el Protocolo de Olivos (PO), vigente desde el 1 de enero de 2004, reglamenta el Sistema de Solución de Controversias del Mercosur ${ }^{75}$. El Protocolo contiene disposiciones relativas a la existencia de procedimientos paralelos con la OMC y otros ACR. En estos casos, el Protocolo admite la opción de foro, al prever que los Estados miembros podrán elegir o convenir el foro de la controversia, pero, una vez iniciado el procedimiento, la misma materia no podrá ser discutida en otros foros ${ }^{76}$.

El sistema abre la posibilidad a los particulares de presentar reclamos sobre medidas legales o administrativas de efecto restrictivo, discriminatorias o de competencia desleal que violen el derecho del MERCOSUR, ante la sección nacional del Grupo Mercado Común del Estado parte donde tenga su residencia habitual o la sede de sus negocios ${ }^{77}$. Las disposiciones sobre procedimiento determinan la realización de consultas con la sección nacional del Grupo Mercado Común del Estado parte al que se atribuye la infracción, y, en la ausencia de una solución en esta fase, la posterior elevación del reclamo al Grupo Mercado Común, que convocará a un grupo de expertos para emitir un dictamen sobre la controversia ${ }^{78}$.

\section{CONSIDERACIONES FINALES}

Los capítulos sobre comercio en los ACR incluyen cada vez más reglas que buscan evitar procedimientos paralelos entre los mismos Estados-parte. Sin embargo, esta estrategia no es observada en las superposiciones entre AIl y las disposiciones sobre solución de controversias inversionista - Estado, y tampoco en las superposiciones en aspectos sustantivos y procesales en derecho comercial. Las partes no son las mismas, y los inversores

74 Véase Fry, J. D. \& Stampalija, J. I. Towards an Agreement on Investment in Mercosur: Conflict and Complementarity of International Investment Law and International Trade-in-Services Law. The Journal of World Investment \& Trade, 2012.

75 Véase Protocolo de Olivos para la Solución de Controversias en el Mercosur. Disponible en internet: http://www.sice.oas.org/trade/mrcsr/olivos/polivosText_s.asp

76 Artículo 1.2, Protocolo de Olivos, supra nota 73.

77 Artículos. 39 y 40, Protocolo de Olivos, supra nota 73.

78 Artículos 40 y siguientes, Protocolo de Olivos, supra nota 73. 
solo pueden obtener un laudo arbitral en virtud de un tratado que permita la solución de controversias entre inversionista - Estado ${ }^{79}$. Por lo tanto, no podemos hablar de un caso típico de forum shopping, como puede ser el caso de un Estado que elige entre varios procedimientos entre diferentes ACR o la OMC. Los inversionistas pueden elegir a menudo entre varios lugares (foros) bajo el mismo AII o utilizar varios AII. Esto puede conducir a "forum shopping" y procedimientos paralelos, pero no hay ningún problema de superposición con otros regímenes de tratados. La OMC y sus órganos (en particular los panelistas y los miembros del Órgano de Apelación) hasta ahora no han estado dispuestos a abordar abiertamente el hecho de que los miembros de la OMC pueden estar unidos por una serie de tratados, y que esto podría afectar a la legislación aplicable en un litigio regido por el ESD de la $\mathrm{OMC}^{80}$.

Además, los mecanismos existentes no son suficientes para abordar el hecho de que varios tribunales arbitrales pueden interpretar el lenguaje y conceptos similares de distintas maneras en virtud de los AII, los ACR y la OMC ${ }^{81}$. Un creciente número de árbitros en la solución de controversias inversionista-Estado está mirando a la jurisprudencia establecida por el Órgano de Apelación de la OMC. Una de las funciones del Órgano de Apelación es crear seguridad jurídica y evitar resultados "incoherentes" ${ }^{12}$. En el Centro Internacional de Arreglo de Diferencias relativas a Inversiones (CIADI), el procedimiento de anulación podría ejercer, en principio, una función similar, aunque en menor medi$\mathrm{da}^{83}$. El proceso de anulación ofrece una opción para reexaminar el laudo original. No obstante, dado que el alcance de la revisión es muy limitado y un órgano de anulación permanente no existe, la creación de un órgano de apelación adecuado que conduciría hacia una mayor coherencia actualmente no es una demanda lejos de concretizarse ${ }^{84}$. Estos factores han contribuido a las críticas dirigidas hacia el CIADI por una falta de coherencia en su jurisprudencia dictada al respeto de la anulación ${ }^{85}$.

79 La cuestión de los procedimientos paralelos entre Estado e inversores no será tema del presente artículo.

80 Véanse las referencias a la jurisprudencia, supra nota 22.

81 Acerca de la discusión sobre el uso de la jurisprudencia sobre trato nacional desarrollada en la OMC y sus referencias por los tribunales de arbitraje inversionista - Estado, véase KURTZ, JürGEN. The Use and Abuse of WTO Law in Investor-State Arbitration: Competition and its Discontents. 20:3 EJIL 20, 749, 2009; Howse, Robert y Chalamish, Efraim. The Use and Abuse of wto Law in Investor-State Arbitration: A Reply to Jürgen Kurtz. 20:4 EJIL 20, 1087, 2009. Sobre consideraciones de los miembros del Comité ad hoc del CIADI, véase también Continental Casualty Company v. The Argentine Republic, ICSID Case n. ${ }^{\circ}$ ARB/03/9, Application for partial annulment, 16 septiembre 2011, par. 89, 90, 108 (h) y 133-134. Sobre el uso de los laudos arbitrales en materia de inversión en el contexto de la OMC véase el Órgano de Apelación de la OMC, las medidas antidumping finales impuestas a EE.UU. sobre el acero inoxidable, Informe de 30 de abril de 2008, WT/DS344/AB/R.

82 Aunque incluso en el sistema de la OMC, los paneles se atreven a diferir a veces, lo que ha sido corregido y criticado por el Órgano de Apelación. Véase QURESHI y ZIEGLER, supra nota 23.

83 Véase Sauvant, Karl P (ed.). Appeals mechanism in international investment disputes. New York: Oxford University Press, 2008.

84 Véase Steger, Debra. Chapter 15. En: De Mestral, Armand y Lévesque, Céline (eds.). Improving International Investment Agreements, supra nota 44, sobre un mecanismo de apelación para inversiones.

85 Véanse los casos sobre Argentina, KIm, Dohyun. The Annulment Committee's Role in Multiplying 
¿Pero qué significa esto con relación a la promoción negociaciones eficientes de AII? Mientras no tengamos un sistema totalmente integrado, donde una institución (posiblemente) con un órgano de apelación -como es el caso de la OMC- juzga todas las disputas económicas (comercio e inversión), no vamos a ser capaces de prevenir este desarro1lo. Incluso en un sistema de derecho económico internacional integrado, las posibles divergencias con otras esferas del derecho internacional (como los derechos humanos, medio ambiente, etc.) prevalecerán. Esto ha sido descrito como la fragmentación y el riesgo de incoherencia que resulta de la existencia de diversos mecanismos de solución de controversias no relacionados en el derecho internacional. Obviamente, un sistema integrado en el que un único órgano permanente se ocupa de las disputas relacionadas con el comercio y las inversiones, posiblemente, puede resolver este problema. Sin embargo, este sistema todavía no se ha desarrollado.

Por el momento, esto puede ser útil para estimular a los árbitros y los miembros de los órganos judiciales de organizaciones multilaterales como la OMC y el CIADI a hacer referencia a ambas jurisprudencias y entablar un debate judicial. Esto podría evitar el escenario donde cada sistema funciona aisladamente $y$, sin duda, aportaría beneficios para el desarrollo de un debate interinstitucional sobre cuestiones específicas que afectan a los flujos mundiales comerciales y de inversión. Al mismo tiempo, la creación de mecanismos plenos de apelación permanentes para los AII, que pueden ser utilizados para impugnar los resultados de los procedimientos arbitrales bajo los AII, pueden ser una opción interesante para probar si esa entidad puede operar de una manera similar al Órgano de Apelación de la OMC, en la ausencia de un acuerdo multilateral y puramente basado en una infinidad de $\mathrm{AII}^{86}$. Los autores acreditan que esto podría hacerse mediante la creación de un órgano adicional bajo el CIADI, preservando los procedimientos de anulación existentes, para permitir que los Estados elijan los mecanismos que consideren apropiados para sus propios AII.

Al mismo tiempo, la situación actual, donde varios órganos (grupos especiales de la $\mathrm{OMC}$, el Órgano de Apelación de la OMC, los tribunales de solución de diferencias en virtud de diversos ACR, los tribunales arbitrales constituidos de acuerdo con diversas normas de procedimiento basados en diferentes AII, comités de anulación del CIADI, los jueces nacionales ${ }^{87}$, etc.) informan al derecho económico internacional no es del todo mala. En primer lugar, parece que hay un creciente intercambio de experiencias entre estos organismos, lo que genera un diálogo judicial. En segundo lugar, todos contribuyen a la jurisprudencia sobre ciertos conceptos jurídicos y nociones, no siempre de manera consistente, pero en general hay cierta consistencia. En tercer lugar, la existencia de re-

Inconsistency in ICSID Arbitration: The Need to Move Away from an Annulment-based System. 86 NYU Law Review 242, 2011.

86 Véase Debra Steger. En: De Mestral, Armand y Lésvesque, Céline (eds.). Improving International Investment Agreements, supra nota 73.

87 Sobre el rol de los jueces nacionales, véase ZiEGLER, ANDrEAs R y BoIE, BerTram. The Relationship between International Trade Law and International Human Rights Law. En: DE WET, ERIKA y VIDMAR, YURE (eds.). Hierarchy in International Law. Oxford: Oxford University Press, 2012. 
sultados divergentes estimula el debate y puede concientizar negociadores acerca de las carencias de sus modelos de tratados o en los marcos existentes ${ }^{88}$. En cuarto lugar, este sistema es muy ambicioso y requiere el análisis sobre la evolución de diversos campos por parte de los académicos, panelistas / árbitros y negociadores. Rastrear y encontrar estos desarrollos no siempre es una tarea fácil (una situación generalmente criticada con relación a las controversias sobre inversiones), pero esto puede ser el precio para una judicialización del derecho económico internacional basada en el imperio de la ley.

ANDREA BJORKLUND y SOPHIE NAPPERT afirman que el área del derecho internacional de las inversiones ofrece el laboratorio perfecto para teorizar sobre las interacciones en la comunicación y su conveniencia. Esta opinión nos parece ser la más apropiada ${ }^{89}$. Esto parece aplicarse también a la superposición entre la OMC, los ACR y los AII, ya que los desarrollos doctrinales en cada área no deberían ser muy aislados, y la ocurrencia de disidencia informada puede dar lugar a un sistema más sofisticado. Incluso, la interpretación de las disposiciones de la OMC o de los ACR por los tribunales arbitrales establecidos en virtud de los AII pueden no poner en peligro la coherencia del sistema existente, siempre que los árbitros involucrados establezcan un diálogo judicial con los grupos especiales, y el Órgano de Apelación y no ignore o abandone completamente el razonamiento de los tribunales anteriores. Desde este punto de vista, no hay ninguna necesidad de cambiar las actuales negociaciones de los capítulos sobre inversión en los ACR o AIl con respecto al funcionamiento de la solución de controversias inversionista - Estado. resultante de estos usado por muchos estados. Véase ZIEGLER, ANDREAS R. The Nascent International Law on Most-Favoured-Nation (MFN) Clauses in Bilateral Investment Treaties (BITs). En: HERRMANN, Christoph y Terhechte, Jörg Philipp (eds.). European Yearbook of International Economic Law. Heidelberg, Germany: Springer, 2010.

89 Véase Bjorklund, Andrea K y Nappert, Sophie. Beyond Fragmentation. uc Davis Legal Studies Research Paper no. 243, 2011. Disponible en internet: SSRN <http://ssrn.com/abstract=1739997>. 